\title{
THE ACTION OF A SOLVABLE GROUP ON AN INFINITE SET NEVER HAS A UNIQUE INVARIANT MEAN
}

\author{
STEFAN KRASA
}

\begin{abstract}
Theorem 1 of the paper proves a conjecture of J. Rosenblatt on nonuniqueness of invariant means for the action of a solvable group $G$ on an infinite set $X$. The same methods used in this proof yield even a more general result: Nonuniqueness still holds if $G$ is an amenable group containing a solvable subgroup $H$ such that $\operatorname{card}(G / H) \leq \operatorname{card}(H)$.
\end{abstract}

1. Introduction. Theorem 1 shows that for the action of a solvable group $G$ on an infinite set $X$ there can never exist a unique $G$-invariant mean on $l^{\infty}(X)$, where $l^{\infty}(X)$ denotes the space of all bounded real valued functions on $X$. A mean $m$ on $l^{\infty}(X)$ is a positive linear functional on $l^{\infty}(X)$ with $m\left(1_{X}\right)=1, m$ is called a "G-invariant mean" if $m(g f)=m(f)$ for every $f \in l^{\infty}(X)$ and for every $g \in G$, where $g f(x)=f\left(g^{-1}(x)\right)$. Theorem 2 is a generalization of Theorem 1 and proves nonuniqueness for the action of an amenable group $G$ on $X$, where $G$ contains a solvable subgroup $H$ such that $\operatorname{card}(G / H) \leq \operatorname{card}(X)$. This paper is largely inspired by [4]. Proposition 6 of [4] shows that if $G$ is nilpotent, then there will always exist more than one $G$-invariant mean on $l^{\infty}(X)$. Additionally it is stated in the same paper [4, p. 531]: "It seems to us very unlikely that Proposition 6 is false if $G$ is solvable, but the same proof does not work". Nonuniqueness need not hold for the general amenable group action as Yang [6] recently showed. This solves a conjecture which is known as "Rosenblatt's problem" in the mathematical literature $[\mathbf{5}$, p. 628]. The proof uses the continuum hypothesis. In Theorem 1 and Theorem 2 even more than nonuniqueness is proved: There exist two disjoint subsets $Y_{1}, Y_{2}$ of $X$ such that $\bigcap_{i=1}^{n} g_{i} Y_{k} \neq \varnothing$ for $k=1,2$ and for every finite subset $\left\{g_{1}, \ldots, g_{n}\right\}$ of $G$. If $G$ does not act transitively on $X$, then there always exist many $G$-invariant means. The methods used in [4] show nonuniqueness if $\operatorname{card}(G) \leq \operatorname{card}(X)$. Obviously there exist examples of solvable groups $G$ such that $\operatorname{card}(G)>\operatorname{card}(X)$ and such that $G$ acts transitively on $X$. Example 3 of [6] even gives an example of a nilpotent group with this property.

EXAMPLE 1. We put $X=\bigoplus_{i=1}^{\infty} Z_{i}$ where $Z_{i}=\mathbf{Z}$ for every $i \in \mathbf{N}$. For $A \subset \mathbf{N}$ we define $\pi_{A}$ as follows. If $x=\left(n_{i}\right)_{i \in \mathbf{N}}$ let $\pi_{A}(X)=\left(m_{i}\right)_{i \in \mathbf{N}}$ where $m_{i}=n_{i}$ if $i \notin A$ and $m_{i}=-n_{i}$ if $i \in A$. For $a \in X$ set $\tau_{a}(x)=a+x$ where addition is defined coordinatewise. Let $G$ be the group generated by $\tau_{a}$ for all $a \in X$ and by $\pi_{A}$ for every $A \subset \mathbf{N}$, then $G$ is solvable and $\operatorname{card}(G)>\operatorname{card}(X)$.

In the proof, we use the following notations: $\operatorname{Per}(X)$ will denote the group of all invertible functions from $X$ onto $X$. For $g \in \operatorname{Per}(X)$ and $Y \subset X$ we put $g Y=\{g(y): y \in Y\}$. For $C \subset \operatorname{Per}(X)$ we put $O_{C}=\left\{C_{x}: x \in X\right\}$ to be the set of

Received by the editors December 18, 1986.

1980 Mathematics Subject Classification (1985 Revision). Primary 43A07. 
all $C$-orbits in $X$. For $F \subset \operatorname{Per}(X)$, and $Y \subset X$, we put $F Y=\{f Y: f \in F\}$. For $A, B \subset \operatorname{Per}(X), A B$ denotes the complex product. $C$ will always denote the set of all cardinals $c$ such that $c \leq \operatorname{card}(X)$.

2. Proof of the nonuniqueness of the mean. It is the essential problem of the following proof that we have to consider group actions where $\operatorname{card}(G)>$ $\operatorname{card}(X)$. The rough idea is to split up the action of $G$ into the action of a finite nested sequence of subsets $P_{i}$ of $\operatorname{Per}(X)$ on $P_{i}$-orbits $A_{i}$ such that $\operatorname{card}\left(P_{i}\right) \leq$ $\operatorname{card}\left(A_{i}\right)$. Then we use the transfinite induction argument of Rosenblatt and Talagrand [4]. Unfortunately, if $\operatorname{card}(X)$ is an uncountable limit cardinal, some problems arise. In this case, we have to choose a family of $P_{i}$-orbits $A_{m}: m \in M$ with increasing cardinality where the index set $M$ is an infinite subset of $C$ with supremum $\operatorname{card}(X)$ such that $\operatorname{card}\left(A_{m}\right)=m$ for every $m \in M$. To each orbit $A_{m}$ there will correspond a subset $Q_{m}$ of $\operatorname{Per}(X)$ such that $\operatorname{card}\left(Q_{m}\right) \leq \operatorname{card}\left(A_{m}\right)$. For each $A_{m}$ and $Q_{m}$ we shall use the transfinite induction argument of [4]. Finally we make a transfinite induction on $M$ to get the desired result. This does not work for an arbitrary $M$. However, if $M$ is a discrete subset of $C$, where $C$ is endowed with the order topology, the argument can be done. It is proved in Lemma 2 that there always exists a discrete subset $M$ of $C$, which is still large enough for our purposes.

Before starting with the proof of Lemma 1, we state the following two well-known equalities for cardinals, we are going to use throughout the proof (cf. [1, p. 128]):

(i) $\aleph_{\alpha}^{n}=\aleph_{\alpha}$ for every ordinal $\alpha$ and for every $n \in N$.

(ii) $\aleph_{\alpha}+\aleph_{\beta}=\aleph_{\alpha} \cdot \aleph_{\beta}=\aleph_{\max (\alpha, \beta)}$ for all ordinals $\alpha, \beta$. We also use standard results about amenable groups (cf. [2]). Throughout we assume that $C$ is endowed with the order topology. We start with a technical lemma.

LEMMA 1. (i) Let $M$ be a subset of $C$ with supremum s. Then there exists a discrete subset $M^{\prime}$ of $M$ with supremum $s$.

(ii) Let $M$ be a discrete subset of $C$. For every $m \in M$ let $N(m)$ be a discrete subset of $C$ with supremum $m$. Then for every $m \in M$ there exists a cardinal $c_{m} \in C$ such that all the sets $N^{\prime}(m)=\left\{n \in N(m): n \geq c_{m}\right\}$ are nonempty and pairwise disjoint and such that $\bigcup_{m \in M} N^{\prime}(m)$ is discrete.

ProOF. (i) We can assume that $s \notin M$ otherwise we would choose $M^{\prime}=\{s\}$. We now construct $M^{\prime}$ via transfinite induction on $M-\{s\}$. Assume that we have constructed for every $c<a, c, a \in M-\{s\}$ a discrete subset $M(c)$ of $M$ such that $M(c)$ contains an element $b$ with $b \geq c$ and such that for $c, c^{\prime} \in M, c<c^{\prime}<a$ implies that $M(c) \subset M\left(c^{\prime}\right)$. We put $N=\bigcup_{c \in M, c<a} M(c)$. If there exists an element $m$ of $N$ such that $m \geq a$ then we put $M(a)=N$ otherwise we choose a cardinal $b \in M$ such that $a<b<s$ and put $M(a)=N \cup\{b\}$. In both cases $M(a)$ is discrete. We define $M^{\prime}=\bigcup_{m \in M, m<s} M(m) . M^{\prime}$ is a discrete subset of $M$ and has $s$ as supremum.

(ii) We use again a transfinite induction: Assume that we have constructed such a net of cardinals $c_{m}$ for every $m \in M$ with $m<a$ where $a \in M$. Let $N=\bigcup_{m \in M, m<a} N(m)$, then $\operatorname{card}(N)<a$, because if $\operatorname{card}(N)=a$ then $a \in \bar{N}$, this together with $a \notin N$ implies that there does not exist a neighborhood $V$ of $a$ such that $V \cap M=\{a\}$ which is a contradiction to the discreteness of $M$. If $a \in N(a)$ we put $c_{a}=a$; otherwise we choose $c_{a}$ such that $\operatorname{card}(N)<c_{a}<a$. 
Definition 1 now defines a cardinality property we are going to need in Definition 2. It is the condition that allows us to use the transfinite induction argument (cf. [4]).

DEFINITION 1. Let $C \subset \operatorname{Per}(X)$ and $Y \subset X$. We say that a set $C^{\prime} \subset \operatorname{Per}(X)$ is $Y$-complete with respect to $C$ if $C^{\prime}$ is finite or $\operatorname{card}\left(C^{\prime}\right) \leq \operatorname{card}(Y)$ and if for every $c \in C$ there exists a $c^{\prime} \in C^{\prime}$ such that $c^{\prime}(y)=c(y)$ for every $y \in Y$.

EXAMPLE 2. Let $A$ be an abelian subgroup of $\operatorname{Per}(X)$ and let $Y$ be an $A$ invariant subset of $X$ such that $A$ acts transitively on $Y$. Then there exists a subset $A^{\prime}$ of $A$ which is $Y$-complete with respect to $A$.

We now give a definition, which we shall explain below.

DEFINITION 2. Let $G$ be a subgroup of $\operatorname{Per}(X)$ and let $C_{i}: i \in\{1, \ldots, n\}, D$ and $E$ be subsets of $\operatorname{Per}(X)$. For every $i \in\{1, \ldots, n\}$ we put $P_{i}=C_{i} C_{i+1} \cdots C_{n} D E$. Furthermore we put $P_{n+1}=D E$ and $P_{n+2}=E$. We say that $\left(C_{i}: i \in\{1, \ldots, n\}\right.$, $D, E)$ is a splitting of $G$ if

(i) $G \subset P_{1}$,

(ii) for every $i \in\{1, \ldots, n+2\}$ and for every $A_{1}, A_{2} \in \mathcal{O}_{P_{i}}, A_{1} \cap A_{2} \neq \varnothing$ implies that $A_{1}=A_{2}$,

(iii) for every $i \in\{1, \ldots, n+2\}$ and for every $A \in O_{P_{i}}$ we have $p A \subset A$ for every $p \in P_{i}$,

(iv) for every $i \in\{1, \ldots, n\}$ and for every $A \in O_{P_{i}}$ there exists a set which is $A$-complete with respect to $C_{i}$,

(v) for every $A \in O_{E}$ and for every $d \in D$, we have $d A \in O_{E}$; and if $d A=d^{\prime} A$ for every $A \in O_{E}$, then $d=d^{\prime}$.

Definition 2 introduces the groups for which we shall prove the nonuniqueness of the mean. Property (i) says that the group action is fully described by the family of subsets of $\operatorname{Per}(X)$, (ii) and (iii) guarantee that we have properly shaped orbits. (iv) is the cardinality property which is necessary for the transfinite induction argument. The sets $D$ and $E$ as well as property (v) will be used for the first time in the proof of Theorem 1, when we show that every solvable group admits a splitting where $D=E=\{e\}$. They are just defined in that way to keep the proof of Theorem 1 short.

LEMMA 2. Let $\left(C_{i}: i \in\{1, \ldots, n\},\{e\},\{e\}\right)$ be a splitting of $G$ where $G$ acts transitively on $X$. Then there exists a $j \in\{1, \ldots, n\}$ and a net of $P_{j}$-orbits $A_{m}: m \in M$ where $M \neq \varnothing$ such that

(i) $M$ is a discrete subset of $C$,

(ii) for every $m \in M$ we have $\operatorname{card}\left(A_{m}\right)=m$,

(iii) for every $i \in\{1, \ldots, j-1\}$ and for every $B \in O_{P_{i}}$ such that $M_{B} \neq \varnothing$ where $M_{B}=\left\{m \in M: A_{m} \subset B\right\}$, we have $\operatorname{card}\left(\bigcup_{m \in M_{B}} A_{m}\right)=\operatorname{card}(B)$,

(iv) for every $m \in M$ there exists a cardinal $c \in C$ such that for every $P_{j+1}$-orbit $B$ which is contained in $A_{m}, \operatorname{card}(B) \leq c<m$ holds.

PROOF. We reformulate Lemma 2 such that an induction argument can be applied.

Claim 1. Let $\left(C_{i}: i \in\{1, \ldots, n\},\{e\},\{e\}\right)$ and $G$ be as above and let $t \in$ $\{1, \ldots, n\}$; then there exists a $j \leq t$ and a net of $P_{j}$-orbits $A_{m}: m \in M$ such that either $j \leq t$ and (i) (iv) of Lemma 2 hold or $j=t$ and (i) (iii) of Lemma 2 hold. 
We prove Claim 1 by induction on $t$ : For $t=1$ there is nothing to prove. We assume therefore that Claim 1 holds for $t<n$ and give the proof for $t+1$.

Let $A_{m}: m \in M$ be the net of $P_{j}$-orbits. We can assume that $j=t$, otherwise we would have the proof for $t+1$. The essential idea of the following is the construction of a net of $P_{t+1}$-orbits $B_{n}: n \in N(m)$ in each $A_{m}$ such that card $\left(B_{n}\right)=n$ for every $n \in N(m)$ and such that $\operatorname{card}\left(\bigcup_{n \in N(m)} B_{n}\right)=m$. Lemma 1(i) and (ii) then complete the proof. The sets $B_{n}: n \in N(m)$ however can only be constructed if Claim 2 from below holds. Claim 3 proves that we can assume without loss of generality that Claim 2 holds.

Claim 2. For every $m \in M$ and for every cardinal $c$ such that $c<m$ there exists a $P_{t+1}$-orbit $B$ such that $B \subset A_{m}$ and $c<\operatorname{card}(B) \leq m$.

Claim 3. Let $M_{1}=\left\{m \in M\right.$ : for every cardinal $c<m$ there exists a $P_{t+1^{-}}$ orbit $B \subset A_{m}$ such that $\left.c<\operatorname{card}(B) \leq m\right\}$ and let $M_{2}=M-M_{1}$. Then for every $k \in\{1, \ldots, t\}$ and for every $P_{k}$-orbit $B$ such that $M_{B} \neq \varnothing$ there exists a net $A_{n}: n \in N$ of $P_{t}$-orbits which are contained in $B$ such that

(i) either $N \subset M^{1}$ or $N \subset M^{2}$,

(ii) for every $i \in\{k, \ldots, t\}$ and for every $P_{i}$-orbit $B^{\prime}$ such that $N_{B^{\prime}} \neq \varnothing$ we have $\operatorname{card}\left(\bigcup_{n \in N_{B^{\prime}}} A_{n}\right)=\operatorname{card}\left(B^{\prime}\right)$.

We prove Claim 3 by induction on $k$. For $k=t$ there is nothing to prove. We now assume that Claim 3 holds for $k>1$ and give the proof for $k-1$.

Let $B$ be a $P_{k-1}$-orbit such that $M_{B} \neq \varnothing$. Then there exists a net $B_{b}: b \in B$ of $P_{k}$-orbits such that $\operatorname{card}\left(B_{b}\right)=b$, such that $M_{B_{b}} \neq \varnothing$ for every $b \in B$ and such that $B$ has $\operatorname{card}(B)$ as supremum. We fix $b \in B$; then there exists a net $A_{n}: n \in N(b)$ such that (ii) holds and such that either $N(b) \subset M^{1}$ or $N(b) \subset M^{2}$. Let $B^{1}$ be the subset of $B$ where $N(b) \subset M^{1}$ and $B^{2}$ the subset of $B$ where $N(b) \subset M^{2}$. Then because $B=B^{1} \cup B^{2}$ either $B^{1}$ or $B^{2}$ has card $(B)$ as supremum in $C$. Lemma $1(\mathrm{i})$ and (ii) applied to that $B^{i}$ yields the existence of a net $A_{n}: n \in N$ such that (i) and (ii) of Claim 3 hold for $B$. This, however, implies Claim 3.

For $k=1$ Claim 3 implies that we can assume for the proof of Claim 1 that Claim 2 holds: The net $A_{n}: n \in N$ fulfils (i)-(iii) of Lemma 2 and also (iv) of Lemma 2 if $N \subset M^{2}$ which would conclude the proof of Claim 1. Therefore we can assume that $N \subset M^{1}$ which means that Claim 2 holds.

We now fix a $m \in M$. Claim 2 and Lemma 1 (i) imply the existence of a net of $P_{t+1}$-orbits $B_{n}: n \in N(m)$ such that $B_{n} \subset A_{m}$ and $\operatorname{card}\left(B_{n}\right)=n$ for every $n \in N(m)$ and such that $N(m)$ is a discrete subset of $C$ with supremum $m$. Lemma 1(ii) now implies the existence of a net $B_{n}: n \in N$ of $P_{t+1}$-orbits such that (i)-(iii) of Lemma 2 hold. This proves Claim 1. For $t=n$ Claim 1 implies Lemma 2.

We are now able to show the nonuniqueness of the mean for amenable groups which have a splitting where $D=E=\{e\}$ by proving the existence of two disjoint subsets $A_{1}, A_{2}$ such that $\bigcap_{i=1}^{n} g_{i} A_{k} \neq \varnothing$ for $k=1,2$ and for every finite subset $\left\{g_{1}, \ldots, g_{n}\right\}$ of $G$. Claim 4 contains the transfinite induction argument of [4]. It is the proof of the induction step of Claim 5. The proof of Claim 5 is the transfinite induction on $M$ which together with Claim 6 yields the complete result.

LEMMA 3. Let $G$ be an amenable subgroup of $\operatorname{Per}(X)$ and let $\left(C_{i}: i \in\{1, \ldots, n\}\right.$, $\{e\},\{e\})$ be a splitting of $G$. If $X$ is an infinite set then there exist many $G$-invariant means on $l^{\infty}(X)$. 
ProOF. We can assume that $G$ acts transitively on $X$ otherwise if $Y_{1}$ and $Y_{2}$ are two different $G$-orbits then there exist two $G$-invariant means $m_{1}$ and $m_{2}$ on $l^{\infty}(X)$ such that $m\left(1_{Y_{k}}\right)=1$ for $k=1,2$ (cf. [2, pp. 16, 17] or [3, Lemma 1]) which implies that $m_{1} \neq m_{2}$.

We can therefore take the net $A_{m}:=m \in M$ of Lemma 2. We are now going to construct to each $A_{m}$ a suitable subset $Q_{m}$ of $\operatorname{Per}(X)$ such that $\operatorname{card}\left(Q_{m}\right) \leq$ $\operatorname{card}\left(A_{m}\right)$ which implies that for each $m \in M$ the transfinite induction argument can be applied and such that a subsequent induction on $M$ yields the complete result.

We now consider the case where $\operatorname{card}(X)>\aleph_{0}$. Let $A_{m}: m \in M$ be the net of $P_{j}$-orbits. We can assume that $m \geq \aleph_{0}$ for every $m \in M$ because the net $A_{m}: m \in M^{\prime}$ where $M^{\prime}=\left\{m \in M: m \geq \aleph_{0}\right\}$ also fulfils (i)-(iv) of Lemma 2.

Let $B^{k}$ be the collection of all $P_{k}$-orbits which contain at least one $A_{m}$; then $B^{1}=$ $\{X\}$ because $G$ acts transitively. Let $H_{X}$ be $X$-complete with respect to $C_{1}$. For every $B \in B^{2}$ we now choose a subset $H_{X}^{B}$ of $H_{X}$ such that $\operatorname{card}\left(H_{X}^{B}\right) \leq \operatorname{card}(B)$, such that $H_{X}^{B_{1}} \subset H_{X}^{B_{2}}$ if $\operatorname{card}\left(B_{1}\right) \leq \operatorname{card}\left(B_{2}\right)$, and such that $\bigcup_{B \in B^{2}} H_{X}^{B}=H_{X}$. We can do that because of (iii) of Lemma 2. Let $H_{B}$ be a $B$-complete subset of $C_{2}$. We put $H_{X, B}=H_{X}^{B} H_{B}$; then $\operatorname{card}\left(H_{X, B}\right) \leq \operatorname{card}(B)$. For $B \in B^{2}$ let $B_{B}^{3}=\left\{B^{\prime} \in B^{3}: B^{\prime} \subset B\right\}$. Now we choose for every $B^{\prime} \in B_{B}^{3}$ a subset $H_{X, B}^{B^{\prime}}$ such that $\operatorname{card}\left(H_{X, B}^{B^{\prime}}\right) \leq \operatorname{card}\left(B^{\prime}\right)$, such that $H_{X, B}^{B_{1}} \subset H_{X, B}^{B_{2}}$ if $\operatorname{card}\left(B_{1}^{\prime}\right) \leq \operatorname{card}\left(B_{2}^{\prime}\right)$, and such that $\bigcup_{B^{\prime} \in B_{B}^{3}} H_{X, B}^{B^{\prime}}=H_{X, B}$ and put $H_{X, B, B^{\prime}}=H_{X,{ }_{B}}^{B^{\prime}} H_{B^{\prime}}$ where $H_{B^{\prime}}$ is $B^{\prime}$-complete with respect to $C^{3}$. We continue this construction until we reach the $P_{j}$-orbits $A_{m}$. We put $Q_{m}=H_{X, B^{2}, B^{3}, \ldots, B^{j-1}, A_{m}}$. Then $\operatorname{card}\left(Q_{m}\right) \leq m$.

If $\operatorname{card}(X)=\aleph_{0}$ the construction is much easier. Instead of considering the net $A_{m}: m \in M$ of Lemma 2 , we choose a $P_{j}$-orbit $B$ where $B$ is countable and where $j$ is the largest index such that a countable orbit exists. We then put $A_{\aleph_{0}}=B$ and choose $A_{m}: m \in\left\{\aleph_{0}\right\}$ as net of $P_{j}$-orbits. Finally we choose for $Q_{\aleph_{0}}$ a subset of $\operatorname{Per}(X)$ which is $A_{\aleph_{0}}$-complete with respect to $C_{1} C_{2} \cdots C_{j}$.

Claim 4. Let $m \in M$ and $W \subset X$ such that $\operatorname{card}(W)<m$. Let $F_{m}=\{F \subset$ $Q_{m}: F$ finite $\}$ and let $F_{\alpha}: \alpha<\delta_{m}$ be an enumeration of $F_{m}$ where $\delta_{m}$ is the least ordinal such that $\operatorname{card}\left(\delta_{m}\right)=\operatorname{card}\left(Q_{m}\right) \leq m$. Then there exists a net of $P_{j+1}$-orbits $P_{\alpha}^{k}: \alpha<\delta_{m}, k=1,2$, which are contained in $A_{m}$ such that all sets $F_{\alpha} P_{\alpha}^{k}: \alpha<\delta_{m}$, $k=1,2$, together with $W$ are pairwise disjoint.

We prove this with transfinite induction. Let $\beta<\delta_{m}$ and assume that we have constructed such a net for every $\alpha<\beta$. We now construct $P_{j+1}$-orbits $P_{\beta}^{1}$ and $P_{\beta}^{2}$. Let $V=W \cup \bigcup_{\alpha<\beta} F_{\alpha} P_{\alpha}^{1} \cup \bigcup_{\alpha<\beta} F_{\alpha} P_{\alpha}^{2}$. Then if $\operatorname{card}(X)=\aleph_{0}$, we get $\operatorname{card}\left(\bigcup_{\alpha<\beta} F_{\alpha} P_{\alpha}^{k}\right)<\aleph_{0}$ for $k=1,2$; and if $\operatorname{card}(X)>\aleph_{0}$, there exists (because of (iv) of Lemma 2) a cardinal $c$ such that $\operatorname{card}\left(P_{\alpha}^{k}\right) \leq c<m$ for every $\alpha<\beta$ and for $k=1,2$, consequently we $\operatorname{get} \operatorname{card}\left(\bigcup_{\alpha<\beta} F_{\alpha} P_{\alpha}^{\bar{k}}\right) \leq \max (c, \operatorname{card}(\beta))<m$ for $k=1,2$. Therefore we get in both cases $\operatorname{card}(V)<m$. Let $F_{\beta}=\left\{q_{1}, \ldots, q_{r}\right\}$, then $\operatorname{card}\left(\bigcup_{i=1}^{r} q_{i}^{-1} V\right)<m$. If $\operatorname{card}(X)=\aleph_{0}, A$ obviously has to contain $\aleph_{0}$-many $P_{j+1}$-orbits, if $\operatorname{card}(X)>\aleph_{0}$ property (iv) of Lemma 2 implies the existence of $m$-many $P_{j+1}$-orbits in $A_{m}$. In both cases there therefore has to exist a $P_{j+1}$-orbit $P_{\beta}^{1}$, such that $P_{\beta}^{1} \subset A_{m}-\bigcup_{i=1}^{r} q_{i}^{-1} V$ which implies that $P_{\beta}^{1} \subset A_{m}$ and that $F_{\beta} P_{\beta}^{1}$, $W$ together with all sets $F_{\alpha} P_{\alpha}^{k}: \alpha<\beta, k=1,2$, are pairwise disjoint. We now put 
$V^{\prime}=V \cup F_{\beta} P_{\beta}^{1}$ and repeat the construction from above to get a $P_{j+1}$-orbit $P_{\beta}^{2}$. This proves Claim 4 .

Claim 5. Let $A_{m}: m \in M$ and $Q_{m}: m \in M$ be as above; then there exist subsets $Y_{1}, Y_{2}$ of $X, Y_{1} \cap Y_{2}=\varnothing$ such that for every $m \in M$ and for every finite subset $\left\{q_{1}, \ldots, q_{r}\right\}$ of $Q_{m}$ there exist $P_{j+1}$-orbits $Z_{1}, Z_{2}$ such that $Z_{1}, Z_{2} \subset A_{m}$ and $\bigcup_{i=1}^{r} q_{i} Z_{k} \subset Y_{k}$ for $k=1,2$.

We prove Claim 5 by induction on $M$. Let $m_{0}$ be the least cardinal of $M$. Let $W=\varnothing$ and $m=m_{0}$. Then Claim 4 implies Claim 5 for $m=m_{0}$ if we put $Y_{k}\left(m_{0}\right)=\bigcup_{\alpha<\delta_{m_{0}}} F_{\alpha} P_{\alpha}^{k}$ for $k=1,2$. We now assume that Claim 5 holds for every $n<m ; n, m \in M$. That means for every $n<m$ there exists a subset $Y_{k}(n)$ of $X, \operatorname{card}\left(Y_{k}(n)\right) \leq n$ such that Claim 5 holds for every finite subset of $Q_{n^{\prime}}$, where $n^{\prime} \leq n$ and that $Y_{k}(n) \subset Y_{k}\left(n^{\prime}\right)$ for $n \leq n^{\prime}<m$ and for $k=1,2$. Let $W=\bigcup_{n<m} Y_{1}(n) \cup \bigcup_{n<m} Y_{2}(n)$; then $\operatorname{card}(W)<m$ because card $\left(Y_{k}(n)\right) \leq n$ for $n \in M, n<m$ and for $k=1,2$ and because there exists a neighborhood $V$ of $m$ such that $V \cap M=\{m\}$ due to the discreteness of $M$. We now use Claim 4 to conclude the proof of Claim 5 .

Claim 6. If there exist subsets $Y_{1}, Y_{2}$ of $X$ such that for every $m \in M$ and for every finite subset $\left\{q_{1}, \ldots, q_{r}\right\}$ of $Q_{m}$ there exist $P_{j+1}$-orbits $Z_{1}, Z_{2} \subset A_{m}$ such that $\bigcup_{i=1}^{r} q_{i} Z_{k} \subset Y_{k}$ for $k=1,2$ then for every finite subset $\left\{h_{1}, \ldots, h_{r}\right\}$ of $P_{1}$ there exist $x_{1}, x_{2} \in X$ such that $\bigcup_{i=1}^{r} h_{i}\left(x_{k}\right) \subset Y_{k}$ for $k=1,2$.

Let $h_{i}=c_{i, 1} c_{i, 2} \cdots c_{i, j} b_{i}$ where $c_{i, k} \in C_{k}$ and $b_{i} \in P_{j+1}$. Then there exists a $c_{i, 1}^{\prime} \in H_{X}$ such that $c_{i, 1}^{\prime}(x)=c_{i, 1}(x)$ for every $x \in X$. There exists a $B \in B^{2}$ such that $c_{i, 1}^{\prime} \in H_{X}^{B}$ for every $i \in\{1, \ldots, r\}$. Let $c_{i, 2}^{\prime} \in H_{B}$ such that $c_{i, 2}^{\prime}(x)=c_{i, 2}(x)$ for every $x \in B$. Then $c_{i, 1}^{\prime} c_{i, 2}^{\prime} \in H_{X, B}$. Therefore there exists a $P_{3}$-orbit $B^{\prime} \subset B$ such that $c_{i, 1}^{\prime} c_{i, 2}^{\prime} \in H_{X, B}^{B^{\prime}}$ for every $i \in\{1, \ldots, r\}$. We continue this construction until we reach a $P_{j}$-orbit $A_{m}$. Then there exists a subset $\left\{q_{1}, \ldots, q_{r}\right\}$ of $Q_{m}$ such that $h_{i}(x)=q_{i} b_{i}(x)$ for every $x \in A_{m}$ and for every $i \in\{1, \ldots, r\}$. Let $Z_{1}, Z_{2}$ be $P_{j+1}$-orbits such that $Z_{1}, Z_{2} \subset A_{m}$ and $\bigcup_{i=1}^{r} q_{i} Z_{k} \subset Y_{k}$ for $k=1,2$, then $q_{i} Z_{k}=c_{i, 1} c_{i, 2} \cdots c_{i, j} Z_{k}$ for $k=1,2$ and $i \in\{1, \ldots, r\}$; and because of (iii) of Definition 2, we get $b_{i} Z_{k} \subset Z_{k}$. This together implies Claim 6 .

Let $Y_{1}, Y_{2}$ be the disjoint subsets of Claim 5 and let $\left\{g_{1}, \ldots, g_{r}\right\}$ be an arbitrary finite subset of $G$. Then $\left\{g_{1}^{-1}, \ldots, g_{r}^{-1}\right\} \subset P_{1}$ and because of Claim 5 and Claim 6 there exist $x_{1}, x_{2} \in X$ such that $\bigcup_{i=1}^{r} g_{i}^{-1}\left(x_{k}\right) \subset Y_{k}$ for $k=1,2$. This however implies that $\bigcap_{i=1}^{r} g_{i} Y_{k} \neq \varnothing$ for $k=1,2$, which implies the existence of two $G$ invariant means $m_{1}$ and $m_{2}$ on $l^{\infty}(X)$ such that $m_{k}\left(1_{Y_{k}}\right)=1$ for $k=1,2$ (cf. [3, Lemma 2]). As $Y_{1}$ and $Y_{2}$ are disjoint $m_{1}$ and $m_{2}$ have to be two different $G$-invariant means.

THEOREM 1. Let $G$ be a solvable group which acts on the infinite set $X$; then there exist many $G$-invariant means on $l^{\infty}(X)$.

PROOF. It is well known that every solvable group is amenable (cf. [2]). Because of Lemma 1 it now remains to prove that there exists a splitting $\left(C_{i}: i \in\right.$ $\{1, \ldots, n\},\{e\},\{e\})$ of $G$.

Claim 7. Let $\left(C_{i}: i \in\{1, \ldots, n\}, D, E\right)$ be a splitting of $G$. Because of (ii) and (v) of Definition 2 we can regard $D$ to be a subset of $\operatorname{Per}\left(O_{E}\right)$. We assume that $D$ is a solvable subgroup of $\operatorname{Per}\left(O_{E}\right)$. Then there exists a splitting $\left(C_{i}: i \in\right.$ $\left.\left\{1, \ldots, n^{\prime}\right\},\{e\}, E\right)$ of $G$. 
We prove Claim 7 by induction on the length of the commutator chain of $D$. For $D=\{e\}$ there is nothing to prove. Assume that Claim 7 holds for every $D$ with a commutator chain of length $k \leq n$ and assume that $D$ has a commutator chain of length $n+1$ that means $D=D_{0} \unrhd D_{1} \unrhd \cdots \unrhd D_{n} \unrhd D_{n+1}=\{e\}$. Then $\left(C_{i}: i \in\{i, \ldots, n\}, D / D_{n},\left[\bigcap_{A \in \mathcal{O}_{E}} T_{D}\left(D_{n} A\right)\right] E\right)$ is a splitting of $G$ where $D / D_{n}$ is considered to be a subgroup of $\operatorname{Per}\left(O_{D_{n} E}\right)$ and where $T_{D}\left(D_{n} A\right)=\{d \in$ $\left.D: d\left(D_{n} A\right)=D_{n} A\right\}$. As Claim 7 holds for $n$ we therefore get a splitting $\left(C_{i}: i \in\right.$ $\left.\left\{1, \ldots, n^{\prime}\right\},\{e\},\left[\bigcap_{A \in O_{E}} T_{D}\left(D_{n} A\right)\right] E\right)$ of $G$ which implies that $\left(C_{i}: i \in\left\{1, \ldots, n^{\prime}\right\}\right.$, $\left.\bigcap_{A \in \mathcal{O}_{E}} T_{D}\left(D_{n} A\right), E\right)$ is a splitting of $G$. We define $H_{1}=\{h \in \operatorname{Per}(X)$ : for every $A \in \mathcal{O}_{D_{n} E}$ there exists $d_{A} \in D_{n}$ such that $d_{A}(x)=h(x)$, for every $\left.x \in A\right\}$. Let $A_{i}: i \in I$ be a collection of $E$-orbits such that each $D_{n} E$-orbit contains exactly one of the sets $A_{i}$. For every $i \in I$ let $S_{i}$ be a system of representatives for the cosets of $T_{D}\left(A_{i}\right) /\left(T_{D}\left(A_{i}\right) \cap D_{n}\right)$ where $T_{D}\left(A_{i}\right)=\left\{d \in D: d\left(A_{i}\right)=A_{i}\right\} . \quad S_{i}$ acts as a solvable group with a commutator chain of length $k \leq n$ on the $E$-orbits contained in $D_{n} A_{i}$. We now define $H_{2}=\left\{h \in \operatorname{Per}(X)\right.$ : for every $i \in I$ there exists a $s_{i} \in S_{i}$ such that $s_{i}(x)=h(x)$ for every $\left.x \in D_{n} A_{i}\right\}$, then $\bigcap_{A \in O_{E}} T_{D}\left(D_{n} A\right) \subset H_{1} H_{2}$. $H_{2}$ can be embedded into the group $\prod_{i \in I} S_{i}$ which is solvable with a commutator chain of length $k \leq n$. $H_{1}$ is an abelian group which acts transitively on every set $D_{n} A_{i}$; therefore Example 2 implies that for every $A \in O_{E}$ there exists a set which is $D_{n} A$-complete with respect to $H_{1}$. We put $C_{n^{\prime}+1}=H_{1}$ then $\left(C_{i}: i \in\right.$ $\left.\left\{1, \ldots, n^{\prime}+1\right\}, H_{2}, E\right)$ is a splitting of $G$, as $H_{2}$ has a commutator chain of length $k \leq n$ there has to exist a splitting $\left(C_{i}: i \in\left\{1, \ldots, n^{\prime \prime}\right\},\{e\}, E\right)$ of $G$ which implies Claim 7.

Obviously $(\{e\}, G,\{e\})$ is a splitting of $G$. Claim 7 implies the existence of a splitting $\left(C_{i}: i \in\{1, \ldots, n\},\{e\},\{e\}\right)$ of $G$ which yields because of Lemma 3 the nonuniqueness of the mean.

REMARK. We could simplify the proof of the result from above a lot, if we assume that $\operatorname{card}(X)$ is no limit cardinal, because then all the nets $A_{m}: m \in M$ could be reduced to nets which contain only one element. Such an assumption, however, would not be justified as it is easy to construct examples where $\operatorname{card}(X)$ is a limit cardinal, $G$ is solvable and $\operatorname{card}(G)>\operatorname{card}(X)$ by similar methods as in Example 1.

We can even prove a slight generalization of Theorem 1.

THEOREM 2. Let $G$ be an amenable group which acts on the infinite set $X$. Let $H$ be a solvable subgroup of $G$ such that $\operatorname{card}(G / H) \leq \operatorname{card}(X)$. Then there exist many $G$-invariant means on $l^{\infty}(X)$.

ProOF. Let $C$ be a system of representatives for the cosets of $G / H$. Then $(C, H,\{e\})$ is a splitting of $G$. Claim 7 and Lemma 3 imply the nonuniqueness.

ACKNOWLEDGMENT. I want to thank F. Hofbauer and H. Rindler for many valuable comments concerning the lucidity of this paper.

\section{REFERENCES}

1. H. Bachmann, Transfinite Zahlen, Ergeb. Math. Grenzgeb., Bd. 1, Springer-Verlag, Berlin and New York, 1967.

2. F. Greenleaf, Invariant means on topological groups, Van Nostrand, New York and London, 1969. 
3. S. Krasa, Non uniqueness of invariant means for amenable group actions, Monatsh. Math. 100 (1985), 121-125.

4. J. Rosenblatt and M. Talagrand, Different types of invariant means, J. London Math. Soc. (3) 24 (1981), 525-532.

5. J. Rosenblatt, Uniqueness of invariant means for measure preserving transformations, Trans. Amer. Math. Soc. 265 (1981), 623-636.

6. Z. Yang, Action of amenable groups and uniqueness of invariant means, preprint.

Department of Mathematics, University of Vienna, A-1090 Vienna, Austria 